\title{
Osteoporosis in patients undergoing lung transplantation
}

\author{
S.L. Ferrari*, L.P. Nicod**, J. Hamacher**, A. Spiliopoulos+, \\ D.O. Slosman++, T. Rochat**, J-P. Bonjour*, R. Rizzoli*
}

Osteoporosis in patients undergoing lung transplantation. S.L. Ferrari, L.P. Nicod, J. Hamacher, A. Spiliopoulos, D.O. Slosman, T. Rochat, J-P. Bonjour, R. Rizzoli. (CERS Journals Ltd 1996.

ABSTRACT: The occurrence of osteoporotic fractures may seriously compromise the quality of life of lung transplant recipients. However, at present, the true risk of osteoporosis in such patients is unknown. We therefore prospectively evaluated bone mass changes in patients undergoing pulmonary transplantation.

Bone mineral density (BMD) of lumbar spine (LS), femoral neck (FN) and femoral shaft (FS), as well as whole body bone mineral content (WB-BMC) were measured in 21 consecutive candidates for lung transplantation $(9$ males and 12 females; mean \pm SD age $47 \pm 11$ yrs). Twelve of the patients had their BMD remeasured within 6 months after surgery, and nine again after 1 year.

Before transplantation, BMD at all sites as well as WB-BMC were significantly decreased as compared to the values in young healthy adults, FN being the most affected (FN $-25 \pm 2 \%$; LS, $-12 \pm 4 \%$; FS $-9 \pm 2 \%$, WB-BMC $-15 \pm 4 \%$ (mean \pm SEM)) Seven out of 20 adult patients $(35 \%)$ fulfilled World Health Organization (WHO) criteria for osteoporosis, i.e. BMD more than 2.5 sD below peak bone mass, whereas three had previously been diagnosed as having osteoporotic fractures of the spine or femoral neck. Within 6 months after transplantation, significant bone loss occurred, mostly at the LS level $(-4.0 \pm 1.7 \%$; $p=0.04)$, despite calcium and vitamin D supplementation. Furthermore, two patients had new osteoporotic vertebral fractures. After 1 year, no further bone loss or new osteoporotic fractures were observed.

In conclusion, evaluation of bone mass and prevention of bone loss should be considered early before lung transplantation. Further studies are needed to determine the efficacy of antiresorptive drugs on the prevention of accelerated bone loss and vertebral fractures after transplantation.

Eur Respir J., 1996, 9, 2378-2382.
*Division of Clinical Pathophysiology, WHO Collaborative Center for Osteoporosis and Bone Disease, Dept of Internal Medicine, **Division of Pneumology, Dept of Internal Medicine, ${ }^{+}$Division of Thoracic Surgery, Dept of Surgery, and ++Division of Nuclear Medicine, Dept of Radiology, University Hospital, 1211 Geneva, Switzerland.

Correspondence: S. Ferrari

Division of Clinical Pathophysiology

Dept of Internal Medicine

University Hospital of Geneva

1211 Geneva 14

Switzerland

Keywords: Corticosteroids

lung transplantation

osteoporosis

vertebral fracture

Received: February 11996

Accepted after revision July 91996

Supported by a grant from the Swiss National Fund for Scientific Research, No 32324 15-91
Organ transplantation has become the ultimate treatment for numbers of patients with terminal failure of kidney, bone marrow, liver, heart, pancreas and, more recently, lung function. Whereas progress in surgery, anaesthesiology, and immunosuppressive therapy has brought about a marked decline in early post-transplantation mortality, quality of life has emerged as a major outcome to evaluate the utility of lung transplantation [1]. Notably, bodily pain has been found to be an important limiting factor of the quality of life in lung-graft recipients [1].

Rapid bone loss and subsequent osteoporotic fractures have been shown to occur after heart, liver or kidney transplantation [2-6]. Patients with chronic lung disease appear to be at high risk of low bone mass because of limited physical activity, poor nutritional status, cigarette smoking and chronic use of corticosteroids [7]. Moreover, lung transplantation may require prolonged immobilization after surgery as well as high doses of corticosteroids to control acute rejection episodes, adding a risk of further bone loss. For these reasons, lung transplant recipients at our institution were supplemented from the time of surgery with calcium and vitamin $\mathrm{D}$, a well-established preventive therapy against steroid-induced osteoporosis [8]. However, the actual risk of osteoporosis in patients undergoing pulmonary transplantation has not yet been properly investigated.

This is the first prospective evaluation of osteoporosis in candidates for lung transplantation, as well as of the early changes of bone mass occurring at the lumbar spine, femoral neck, femoral shaft and total body in lung transplant recipients.

\section{Methods}

\section{Study subjects}

Between December 1992 and September 1995, we evaluated 21 consecutive patients with end-stage respiratory failure for lung transplantation, of whom 14 were then transplanted during this period. One died 3 months after surgery and two others 9 months after surgery. The immunosuppressive regimen included one peroperative pulse of methylprednisolone $(500 \mathrm{mg})$, 
followed by the administration of antilymphocytic antibodies (ATGAM®; Upjohn; $15 \mathrm{mg} \cdot \mathrm{kg}^{-1}$ q.d.) for 1 week. Maintenance therapy included oral prednisone $(0.2$ $\mathrm{mg} \cdot \mathrm{kg}^{-1}$ q.d. for 1 week, $0.5 \mathrm{mg} \cdot \mathrm{kg}^{-1}$ q.d. for 3 months, and then progressive lowering to $0.2 \mathrm{mg} \cdot \mathrm{kg}^{-1} q . d$. at 5 months), azathioprine (50-100 $\mathrm{mg}$ q.d.) and cyclosporin (blood trough levels, 200-400 $\mathrm{ng} \cdot \mathrm{mL}^{-1}$ ). Acute rejection episodes were treated with $0.5-1 \mathrm{~g}$ q.d. of intravenous methylprednisolone for three consecutive days, followed by an increase of usual immunosuppressive drugs. In the absence of a satisfactory response, patients were then treated with antithymocyte globulin injections.

\section{Study design}

Bone mass was first measured in the 21 candidates for lung transplantation during their pretransplantation examination. It was remeasured at the time of transplantation when the examination had been performed more than 6 months before surgery. Bone mass was then reevaluated within 3-6 months after surgery in 12 patients, and again at 1 year in nine patients. Antiosteoporosis preventive therapy was prescribed to all patients from the time of transplantation as an oral supplementation of calcium (1 g q.d.) and vitamin D (1,000 I.U. q.d.) [8]. One amenorrhoeic woman also received hormone replacement therapy (HRT) (2 mg q.d. oestradiol, $1 \mathrm{mg}$ q.d. oestriol, and $1 \mathrm{mg}$ q.d. norethisterone acetate). After 3-6 months, HRT or sodium monofluorophosphate $(26 \mathrm{mg}$ fluoride ion q.d.) were prescribed to five patients with significant bone loss and/or new osteoporotic fractures.

\section{Bone mass measurement}

Bone mineral density (BMD $\mathrm{g} \cdot \mathrm{cm}^{-2}$ ) of lumbar spine (LS), femoral neck (FN) and femoral shaft (FS), as well as whole body bone mineral content (WB-BMC; normalized for body height, $\left.\mathrm{g} \cdot \mathrm{m}^{-1} \times 1.70\right)$, were measured by dual energy X-ray absorptiometry (DXA), (Hologic QDR 1000W and QDR 2000). The coefficient of variation of DXA was $1-2 \%$ or less for regional BMD as well as for WB-BMC measurements [9, 10]. Bone mass values at baseline were expressed as percentages of peak bone masses in sex-matched young healthy adults, which were: LS BMD $1.07 \pm 0.10$ and $1.06 \pm 0.13 \mathrm{~g} \cdot \mathrm{cm}^{-2}$; FN BMD $0.90 \pm 0.11$ and $0.96 \pm 0.15 \mathrm{~g} \cdot \mathrm{cm}^{-2}$; FS BMD $1.73 \pm 0.17$ and $2.02 \pm 0.13 \mathrm{~g} \cdot \mathrm{cm}^{-2}$; WB BMC, $2259 \pm 248$ and $2573 \pm 327 \mathrm{~g} \cdot \mathrm{m}^{-1} \times 1.70($ mean $\pm \mathrm{sD})$, in females and males, respectively $[9,10]$. Bone mass changes during follow-up were expressed as percentage difference from baseline.

New vertebral fractures were diagnosed when clinical symptoms compatible with the diagnosis were present together with decreased vertebral body height $(\geq 20 \%)$ on conventional X-rays and/or with evidence of vertebral fracture and oedema on magnetic resonance imagery.

\section{Analysis}

The statistical significance of the results was assessed by two-tailed Student's t-test or Pearson correlation coefficient of simple linear regression. A p-value of less than 0.05 was considered significant.

\section{Results}

The mean \pm SD age of 21 candidates to lung transplantation (9 males and 12 females) was $47 \pm 11$ yrs (range 32-65 yrs) (table 1). The most frequent causes for terminal respiratory failure included: chronic obstructive pulmonary disease (COPD), in 13 patients; idiopathic

Table 1. - Patients characteristics

\begin{tabular}{|c|c|c|c|c|c|c|}
\hline $\begin{array}{l}\text { Patient } \\
\text { No. }\end{array}$ & Sex & $\begin{array}{l}\text { Age } \\
\text { yrs }\end{array}$ & Diagnosis & OP risk factors & Anti-OP treatment & $\begin{array}{c}\mathrm{BMI} \\
\mathrm{kg} \cdot \mathrm{m}^{-2}\end{array}$ \\
\hline 1 & M & 43 & COPD & Steroids, smoking & - & 18.7 \\
\hline 2 & M & 65 & IPF & Steroids & - & 16.8 \\
\hline 3 & M & 46 & COPD & Smoking & - & 19.8 \\
\hline 4 & M & 55 & COPD & Smoking & - & 24.5 \\
\hline 5 & $\mathrm{~F}$ & 11 & $\mathrm{CF}$ & Growth retardation & - & 13.4 \\
\hline 6 & $\mathrm{M}$ & 32 & $\mathrm{CF}$ & - & $\mathrm{Ca}+$ vit $\mathrm{D}$ & 19.6 \\
\hline 7 & $\mathrm{~F}$ & 38 & COPD & Steroids, smoking & $\mathrm{Ca}+$ vit $\mathrm{D}$ & 15.1 \\
\hline 8 & $\mathrm{~F}$ & 47 & COPD & Steroids, smoking, menopause & - & 20.9 \\
\hline 9 & $\mathrm{~F}$ & 43 & $\mathrm{CF}$ & Steroids & - & 15.4 \\
\hline 10 & M & 49 & IPF & Steroids, smoking & $\mathrm{Ca}+$ vit $\mathrm{D}+$ fluoride & 26.2 \\
\hline 11 & $\mathrm{~F}$ & 50 & IPF & Steroids, menopause & $\mathrm{Ca}+$ vit $\mathrm{D}$ & 28.3 \\
\hline 12 & $\mathrm{~F}$ & 45 & COPD & - & $\mathrm{Ca}$ & 19.5 \\
\hline 13 & $\mathrm{~F}$ & 55 & COPD & Steroids, smoking, menopause & HRT & 29.0 \\
\hline 14 & M & 56 & COPD & Steroids, smoking, OP VF & - & 23.9 \\
\hline 15 & M & 51 & COPD & Steroids, smoking, OP VF & - & 25.1 \\
\hline 16 & M & 47 & COPD & Steroids, smoking & $\mathrm{Ca}+$ vit $\mathrm{D}$ & 31.3 \\
\hline 17 & $\mathrm{~F}$ & 48 & COPD & Smoking, menopause & HRT & 18.7 \\
\hline 18 & $\mathrm{~F}$ & 54 & COPD & Smoking, menopause & HRT & 19.8 \\
\hline 19 & $\mathrm{~F}$ & 49 & COPD & Steroids, smoking, OP FNF & $\mathrm{Ca}+$ vit $\mathrm{D}$ & 27.1 \\
\hline 20 & $\mathrm{~F}$ & 40 & LLM & Smoking, amenorrhoea & Progestagens & 17.0 \\
\hline 21 & $\mathrm{~F}$ & 56 & PPHT & - & - & 20.3 \\
\hline
\end{tabular}

M: male; F: female; OP: osteoporosis; BMI: body mass index; COPD: chronic obstructive pulmonary disease; CF: cystic fibrosis; IPF: idiopathic pulmonary fibrosis; PPHT: primary pulmonary hypertension; LLM: lymphangioleiomyomatosis; VF: vertebral fracture; FNF: femoral neck fracture; Ca + vit D: calcium and vitamin D supplements; HRT: hormone replacement therapy with oestroprogestagens. 


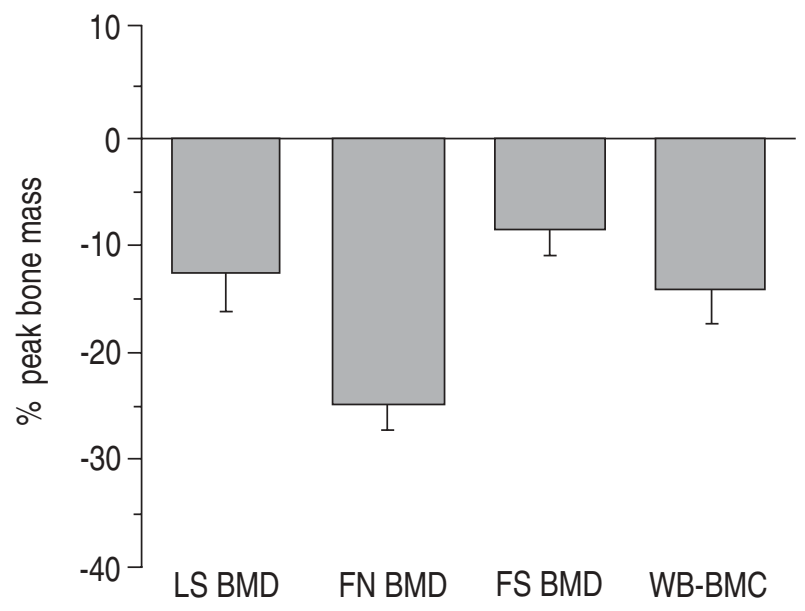

Fig. 1. - Bone mineral density of lumbar spine (LS BMD), femoral neck (FN BMD), femoral shaft (FS BMD), and whole body bone mineral content (WB-BMC) in 20 adults candidates for lung transplantation. The results, expressed as percentage differences ( \pm SEM) from peak bone mass in sex-matched young healthy adults $[9,10]$, are statistically significant at all sites $(\mathrm{p}<0.01)$.

pulmonary fibrosis (IPF), in 3 patients; and cystic fibrosis $(\mathrm{CF})$, in 2 patients. Other common risk factors for osteoporosis were: cigarette smoking (14 patients); chronic corticosteroid treatment (12 patients); and menopause or amenorrhoea (6 patients). Nine patients were receiving antiosteoporosis therapy with calcium and vitamin D or oestroprogestagens, because of chronic corticosteroid treatment, menopause or established osteoporosis.

Among the 20 adult patients, FN BMD and LS BMD, as well as WB-BMC, were markedly decreased as compared to the peak bone mass in healthy young adults $(-25 \pm 2 \% \quad(\mathrm{p}=0.0001) ;-12 \pm 4 \% \quad(\mathrm{p}=0.003) ;$ and $-15 \pm 4 \%$ $(\mathrm{p}=0.002)$, respectively $($ mean \pm SEM $)$, whereas FS BMD was less affected $(-9 \pm 2 \% ; \mathrm{p}=0.001)$ (fig. 1). Overall, $60 \%$ of the candidates for lung transplantation had regional BMD more than 2.0 SD below peak bone mass. Seven out of 20 adult subjects $(35 \%)$ met WHO criteria for osteoporosis, i.e. BMD more than 2.5 sD below peak bone mass [11], and three had previously sustained osteoporotic fractures of the spine or proximal femur. In addition, we also investigated an 11 year old female with $\mathrm{CF}$, who is not included in the above analysis, and who was found to have a 2 year delay in statural growth and bone age. FN BMD and LS BMD were both $18 \%$ lower than in age-matched controls.

LS BMD appeared somewhat lower in male than female adults $(-18 \pm 4$ vs $8 \pm 5 \%$, respectively; $\mathrm{p}=0.15)$. No such trend was found for bone mass measurements at the other sites. However, there was a nonsignificant trend for lower LS BMD and FN BMD among women with menopause or amenorrhoea as compared to women with regular menses (LS $-12 \pm 4$ vs $-1 \pm 10 \%$; FN $-28 \pm 3$ vs $-19 \pm 5 \%$ ). By contrast, there was no apparent BMD difference according to corticosteroids use, smoking status or antiosteoporosis therapy, nor was body mass index (BMI) correlated with bone mass at any site (data not shown).

Twelve out of 14 lung transplant recipients ( 7 males and 5 females) had a second measurement of regional BMD and WB-BMC 3-6 months after surgery (mean \pm SD,
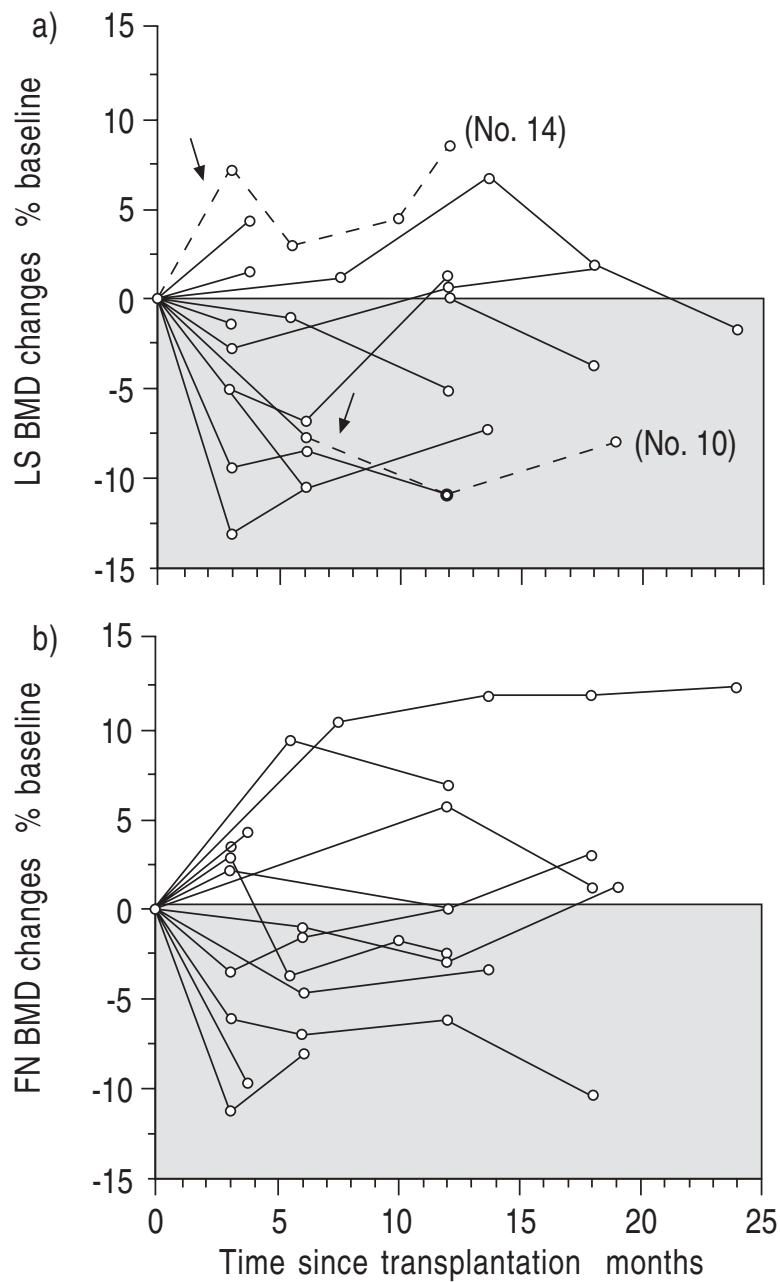

Fig. 2. - Individual changes in bone mineral density (BMD) of: a) lumbar spine (LS); and b) femoral neck (FN) after lung transplantation in 12 patients. Results are expressed as percentage difference from baseline. The broken lines indicate LS measurements made in two patients (patient number in parenthesis) after the occurrence of oesteoporotic fractures (arrows) in the area of interest.

$4.5 \pm 1.6$ months). Bone loss was detected in eight of them at the level of the LS and in seven at the FN (fig. 2 ). As compared to baseline, mean bone mass was significantly decreased at the LS level $(-4.0 \pm 1.7 \%$; $p<0.05)$, but not at the other skeletal sites (table 2). Changes in BMD were similar in both sexes, and did not correlate with BMD at baseline (data not shown). By contrast,

Table 2. - Mean bone mass changes after lung transplantation

\begin{tabular}{lcr}
\hline & \multicolumn{2}{c}{ Bone mass } \\
& $\leq 6$ months & $\begin{array}{c}\text { baseline } \\
1 \text { year }\end{array}$ \\
\hline Lumbar spine & $-4.0 \pm 1.7 *$ & $-2.1 \pm 2.3$ \\
Femoral neck & $-0.8 \pm 2.0$ & $+1.0 \pm 1.9$ \\
Femoral shaft & $-1.0 \pm 0.8$ & $-1.1 \pm 0.4$ \\
Whole body & $-0.5 \pm 0.7$ & $+2.3 \pm 1.5$
\end{tabular}

Values are presented as mean \pm SEM: Twelve patients had their bone mass assessed $\leq 6$ months after transplantation and nine after 1 year. One LS BMD measurement at $\leq 6$ months and 2 at one year were withdrawn because of osteoporotic fractures in the region of measurement. LS: lumbar spine; BMD bone mineral density. ${ }^{*}: \mathrm{p}<0.05$, as compared to baseline. 
a correlation of borderline significance was observed between LS bone loss and the cumulative dose of corticosteroids administered during this period $(\mathrm{r}=0.56$; $\mathrm{p}=0.09$ ). This correlation became highly significant after one patient receiving HRT at that time was removed $(\mathrm{r}=0.86 ; \mathrm{p}=0.003)$.

Two male patients had multiple osteoporotic vertebral fractures and another male had a traumatic fracture of the pelvis during this period. In the two former, LS BMD before transplantation was $15 \%$ lower than peak bone mass, and one of them had a rapid bone loss of $8 \%$ documented shortly before the vertebral fractures occurred (fig. 2).

In the nine patients ( 5 males and 4 females) who could be re-evaluated 1 year after transplantation, no significant bone loss was observed as compared to their earlier measurements (fig. 2 and table 2). On the contrary, there was a trend for increased regional BMD and WB$\mathrm{BMC}$ in these patients. Importantly, at that time, three females had been receiving oestrogens and two males sodium monofluorophosphate for $7.2 \pm 1.5$ months, in addition to calcium and vitamin $\mathrm{D}$.

\section{Discussion}

This study demonstrates a high prevalence of osteoporosis (35\%, according to WHO criteria), as well as several cases of previous osteoporotic fractures, in candidates for lung transplantation. Similar observations have been reported previously in candidates for heart or liver transplantation $[2,3]$. Although none of the multiple well-established risk factors for osteoporosis which were present in our cohort, such as prior smoking, low BMI, amenorrhoea and chronic corticosteroid therapy, could be associated with a significantly decreased BMD (possibly due to a limited number of patients), they probably contributed altogether to an increased risk for osteoporosis [7]. Interestingly, the lowest BMD values were measured in males and amenorrhoeic/postmenopausal women, which is compatible with a major protective role of oestrogens against bone loss. By contrast, less than half the patients were receiving osteoporosis preventive therapy before transplantation. These observations emphasize the need for an early evaluation of bone status and the administration of anti-osteoporosis preventive therapy in all candidates for pulmonary transplantation, and possibly in the majority of patients with chronic respiratory failure.

According to the widely-accepted recommendations concerning antiosteoporosis preventive therapy with calcium and vitamin D in patients undergoing longterm corticosteroid treatment [8], all of the lung transplant recipients in this study received such supplements from the time of surgery. Nevertheless, rapid bone loss occurred within 6 months after transplantation, mainly affecting the lumbar spine, a site rich in trabecular bone. Postsurgical immobilization and immunosuppressive therapy, including high-dose pulse corticosteroids used to treat early rejection episodes, are likely to have contributed to this evolution. Indeed, a significant correlation between the rate of LS bone loss and the cumulative dose of corticosteroids was observed in all patients, with the exception of one on HRT. Meanwhile, osteoporotic vertebral fractures were documented in two male patients. In one of these, fractures occurred as he resumed intense physical activity, which was made possible by excellent lung graft function. At the present time, only one case of multiple vertebral fractures appearing shortly after lung transplantation has been reported [12]. The consequences of vertebral fractures in lung graft recipients are still unknown. However, thoracic vertebral fractures may cause bone pain and lead to chest wall deformities, thereby compromising lung graft function. Thus, osteoporosis might severely impair the quality of life in lung transplant recipients [1].

Interestingly, early bone loss was not detectable in a woman on oestrogen replacement therapy given immediately after transplantation, despite the administration of a large cumulative dose of corticosteroids $(9,900 \mathrm{mg}$ prednisone equivalent). This observation further suggests that oestrogens, which are an effective treatment against glucocorticoid-induced osteoporosis [13], must be considered as a first-line preventive therapy in amenorrhoeic patients undergoing lung transplantation. Alternatively, bisphosphonates, which have also been shown to prevent bone loss in patients on long-term steroid treatment [14], need to be investigated in this setting.

Between 6 months and 1 year after transplantation, bone mass was apparently stable, or even improved, at all sites. Notably, three women were receiving HRT during that period, whereas two men were on monofluorophosphate [15]. Although these treatments are likely to have contributed to the favourable outcome of the group, it may be postulated that lung transplantation by itself corrected many risk factors for osteoporosis, including poor oxygenation, diminished exercise capacity and low BMI. Under these conditions, it appears most important to prevent early bone loss in lung transplant recipients, in order to decrease the incidence of osteoporotic fractures occurring soon after surgery.

In conclusion, osteoporosis was frequently diagnosed among candidates to lung transplantation, and the risk of skeletal damage was high shortly after surgery. Therefore, early evaluation of bone mass and efficient prevention of bone loss should be considered in all patients undergoing lung transplantation.

\section{References}

1. Gross CR, Savik K, Bolman RM, Hertz MI. Long-term health status and quality of life outcomes of lung transplant recipients. Chest 1995; 108: 1587-1593.

2. Muchmore JS, Cooper DKC, Ye Y, Schlegel VT, Zuhdi $\mathrm{N}$. Loss of vertebral bone density in heart transplant patients. Transplant Proc 1991; 23:1183-1185.

3. McDonald JA, Dunstan CR, Dilworth P, et al. Bone loss after liver transplantation. Hepatology 1991; 14: 613-619.

4. Julian BA, Laskow DA, Dubovsky J, Dubovsky EV, Curtis JJ, Quarles LD. Rapid loss of vertebral mineral density after renal transplantation. N Engl J Med 1991; 325: 544-550.

5. Shane E, Rivas MDC, Silverberg SH, Kim TS, Staron RB, Bilezikian JP. Osteoporosis after cardiac transplantation. Am J Med 1993; 94: 257-264.

6. Lee AH, Mull RL, Keenan GF, Callegari PE, et al. Osteoporosis and bone morbidity in cardiac transplant recipients. Am J Med 1994; 96: 35-41. 
7. Dempster DW, Lindsay R. Pathogenesis of osteoporosis. Lancet 1993; 341: 797-805.

8. Meunier P. Is steroid-induced osteoporosis preventable? New Engl J Med 1993; 328: 1781-1782.

9. Slosman DO, Rizzoli R, Pichard C, Donath A, Bonjour JP. Longitudinal measurement of regional and whole body bone mass in young healthy adults. Osteoporosis Int 1994; 4: 185-190.

10. Slosman DO, Casez JP, Pichard C, et al. Assessment of whole-body composition with dual-energy X-ray absorptiometry. Radiology 1992; 185: 593-598.

11. WHO Study Group. Assessment of fracture risk and its application to screening for postmenopausal osteoporosis. WHO Technical Report Series 843, Geneva 1994.

12. Day JD, Walden SM, Stuart SR, Hutchins GM. Hruban
RH. Fatal fat embolism syndrome after numerous vertebral body compression fractures in a lung transplant recipient. J Heart Lung Transplant 1994; 13: 785-790.

13. Lukert BP, Johnson BE, Robinson RG. Estrogen and progesterone replacement therapy reduces glucocorticoid-induced bone loss. J Bone Miner Res 1992; 7: 1063-1069.

14. Reid IR, King AR, Alexander CJ, Ibbertson HK. Prevention of steroid-induced osteoporosis with (3-amino- 1-hydroxypropylidene)-1 bisphosphonate (APD). Lancet 1988; i: 143-146.

15. Rizzoli R, Chevalley T, Slosman D, Bonjour JP. Sodium monofluorophosphate increases lumbar spine bone mineral density in corticosteroid-induced osteoporosis. Osteoporosis Int 1995; 5: 39-46. 